IDDF2018-ABS-0199 GUT MICROBIOME PROFILING OF MALAYSIANS: A SNAPSHOT

${ }^{1}$ Hui-min Neoh*, ${ }^{1}$ Muhammad Afiq Osman, ${ }^{1}$ Nurul Syakima Ab Mutalib, ${ }^{1}$ Siok Fong Chin, ${ }^{1}$ Mia Yang Ang, ${ }^{2}$ Luqman Mazlan, ${ }^{3}$ Chai Soon Ngiu, ${ }^{1}$ Rahman Jamal. ${ }^{1}$ UKM Medical Molecular Biology Institute (UMBI), Universiti Kebangsaan Malaysia, Malaysia; ${ }^{2}$ Department of Surgery, Faculty of Medicine, Universiti Kebangsaan Malaysia, Malaysia; ${ }^{3}$ Department of Medicine, Faculty of Medicine, Universiti Kebangsaan Malaysia, Malaysia

\subsection{6/gutjnl-2018-IDDFabstracts.27}

Background A balanced commensal gut microbiome is believed to be important to maintain a healthy digestive system, where disruption of this balance or dysbiosis have been associated with diseases such as metabolic syndromes, neurologic disorders and also cancer. Gut microbiome profiling studies of various populations in the world showed that most populations carry the Bacteroides, Prevotella, or Ruminococcus genera in abundance. We carried out a study to determine the gut microbiome profile of Malaysians, which consists of individuals from largely Malay, Chinese or Indian heritage.

Methods Gut mucosal tissues were obtained from subjects undergoing colonoscopy screening in an endoscopy clinic in UKM Medical Centre, Kuala Lumpur, Malaysia, in 20142017. DNA extraction was performed using a QIAGEN DNA Micro kit; 16S rRNA amplicon sequencing on the V3/V4 regions was carried out for all samples using an Illumina MiSeq sequencer. Forward and reverse sequences of each sample were assembled using SeqPrep and later analysed using QIIME, where assembled reads were clustered into Operational Taxonomic Units (OTUs) and aligned against the GreenGenes 16S database.

Results A total of 15 subjects were included in the study. Mean age of the subjects was 54.4 and they were mostly of either Malay $(n=11,61.1 \%)$ or Chinese $(n=6,33.3 \%)$ heritage. A total of 17 phyla and 274 genera were detected in our subjects, where the top three prevalent phyla were Firmicutes, Bacteroidetes, and Fusobacteria; Bacteroides, Fusobacterium and Faecalibacterium were the top three prevalent bacteria genera. Interestingly, no difference in alpha diversity was observed between genders and ethnicities.

Conclusions This study presents a snapshot of the Malaysian gut microbiome. At a glance, it seems that bacteria genera abundance of the Malaysian gut microbiome might be slightly different from other populations of the world, with a higher abundance of the CRC-associated Fusobacterium genus found in the general Malaysian population. Nevertheless, this observation has to be reconfirmed in a larger study.

\section{IDDF2018-ABS-0207 WINNING THE WAR AGAINST COLON CANCER: CHEMO-PREVENTIVE POTENTIAL OF NOVEL STREPTOMYCES SPECIES DERIVED FROM MANGROVE FOREST IN MALAYSIA}

${ }^{1}$ Learn-Han Lee*, ${ }^{1}$ Hooi-Leng Ser, ${ }^{2}$ Nurul-Syakima Ab Mutalib, ${ }^{1}$ Tahir Mehmood Khan, ${ }^{3}$ Kok-Gan Chan, ${ }^{4}$ Bey-Hing Goh. ${ }^{1}$ Novel Bacteria and Drug Discovery Research Group, School of Pharmacy, Monash University Malaysia, Malaysia; ${ }^{2}$ UKM Medical Molecular Biology Institute (UMBI), UKM Medical Centre, University Kebangsaan Malaysia, Malaysia; ${ }^{3}$ Division of Genetics and Molecular Biology, Institute of Biological Sciences, Faculty of Science, University of Malaya, Malaysia; ${ }^{4}$ Biofunctional Molecule Exploratory Research Group, School of Pharmacy, Monash University Malaysia, Malaysia

\subsection{6/gutjnl-2018-IDDFabstracts.28}

Background Chemoresistance remains a major challenge in cancer therapy including colon cancer (CC). Researchers have been searching for efficient, potent drug candidates to combat against CC. Microorganisms are 'mini-factories' for bioactive compounds that can be exploited as therapeutic agents. The members of the genus Streptomyces is well-known as prolific

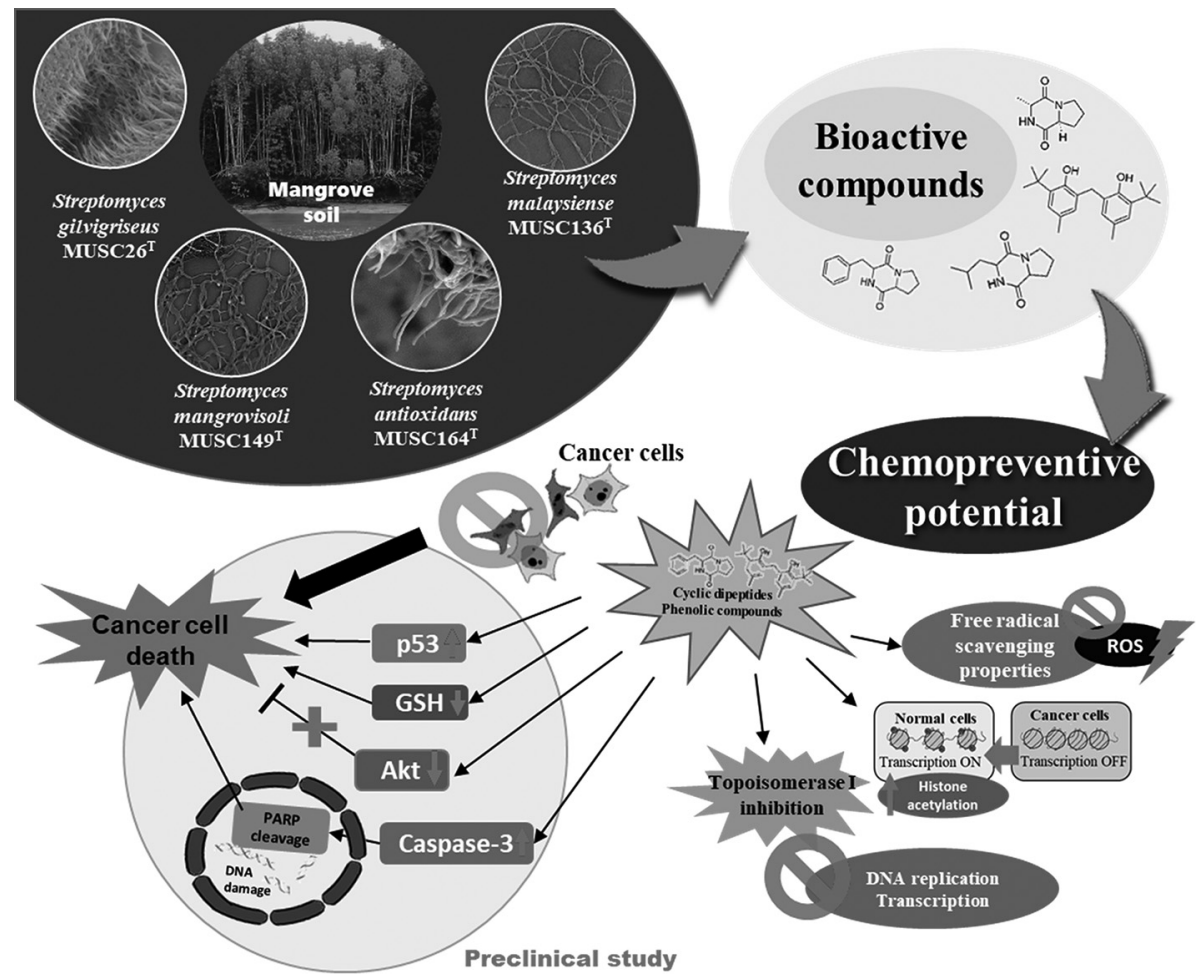

Abstract IDDF2018-ABS-0207 Figure 1 Chemopreventive potential of novel, mangrove-derived streptomycetes against colon cancer 